\title{
Pauli blocking effects in thermalization of relativistic plasma
}

\author{
M. A. Prakapenia \\ ICRANet-Minsk, B. I. Stepanov Institute of Physics, \\ National Academy of Sciences of Belarus, 220072 Nezaležnasci Av. 68-2, Minsk, Belarus and \\ Department of Theoretical Physics and Astrophysics, \\ Belarusian State University, 220030 Nezaležnasci Av. 4, Minsk, Belarus \\ G. V. Vereshchagin \\ ICRANet, 65122 Piazza della Repubblica, 10, Pescara, Italy and \\ INAF Istituto di Astrofisica e Planetologia Spaziali, \\ 00133 Via del Fosso del Cavaliere,100, Rome, Italy
}

\begin{abstract}
We investigate the effects of Pauli blocking on thermalization process of relativistic plasma by solving relativistic Uehling-Uhlenbeck equations with QED collision integral for all binary and triple processes. With this purpose we consider nonequilibrium initial state of plasma to be strongly degenerate. We found that when electron-positron annihilation is active, initial plasma degeneracy is quickly destroyed. As a result in a wide range of final temperatures ranging from nonrelativistic to mildly relativistic $0.1 m_{e} c^{2} \leq k_{B} T \leq 10 m_{e} c^{2}$ thermalization is not affected by Pauli blocking. Conversely, when electron-positron annihilation process is inactive, thermalization process in such degenerate plasma is strongly affected by Pauli blocking. This is possible either in a nonrelativistic plasma, with equilibrium temperature $k_{B} T \leq 0.3 m_{e} c^{2}$, or in photon-electron plasma. In these cases all reaction rates are strongly suppressed by Pauli blocking and thermalization does not occur until electrons can populate energy states above the Fermi energy. Soon after this happens thermalization proceeds suddenly in an avalanche-like process.
\end{abstract}

\section{INTRODUCTION}

Pauli exclusion principle is a fundamental principle of quantum mechanics and it manifests in many branches of physics: condensed matter, chemistry, molecular biology, etc. Such phenomena as insulator-conductor transition or Mott effect, Feshbach resonance and atom-exchange chemical reactions [1 5] deal with Pauli blocking. Pauli principle plays a crucial role in a dense matter state 6, it affects conductivity in a dense Coulomb plasma [7, for extreme plasma densities it leads to depression of ionization potential [8, it is crucial in many nuclear physics problems 912 . The development of new techniques for atomic gases cooling allows to create Fermi gases in a laboratory. Suppression of interactions in such a degenerate Fermi gases is subject of active research [13 16]. Lattice Fermi gas can serve as direct probe of Pauli blocking 17. Existence and stability of such compact astrophysical objects as white dwarfs [18, 19] and neutron stars is possible due to Pauli blocking [20, which modifies the equation of state at high densities and prevents gravitational collapse of stars with mass below $\sim 3$ solar masses after their nuclear fuel is exhausted.

Another arena of manifestation of the Pauli blocking effect is relativistic plasma. In addition to astrophysical environments, it is also of interests in laboratory experiments targeting observation of QED processes 21 . Attempts to create electron-positron plasma with high power lasers or laser interactions with matter $22+25$ are under way. Such plasma is typically formed out of equilibrium. Thermalization process of non-equilibrium relativistic plasmas has been studied [26, 27, and thermalization timescales were determined [28, but so far Pauli blocking in this process has been neglected.

Kinetic equations accounting for Pauli blocking and Bose enhancement effects are called Uehling-Uhlenbeck equations. These equations correctly describe the limit of fully degenerate state, when Pauli blocking reduce reaction rates of fermions to zero. The present work deals with optically thick relativistic plasma composed of electron, positrons and photons [29] and focuses on strongly degenerate initial plasma states. The paper is organized as follows. In Section II conditions of plasma degeneracy are reviewed. In Section III relativistic Boltzmann equations are introduced. In Section IV thermalization process of superdegenerate plasma is discussed. The main results are summarized in Section V. In appendix we present a table describing Bose enhancement and Pauli blocking factors for all binary and triple reactions considered.

\section{FERMION CRITICAL DENSITY}

The degree of plasma degeneracy is characterized by the parameter 30 .

$$
D=\frac{1}{n \lambda_{t h}^{3}},
$$

where $n$ is number density of particles in plasma, $\lambda_{t h}=$ $\frac{c \hbar}{k T}$ is the thermal wave-length, $k$ is Boltzmann constant, $T$ is temperature, $\hbar=h /(2 \pi), h$ is Planck constant. The number density - energy density diagram for relativistic electron-positron plasma is presented in Fig. 1. The black line corresponds to $D=1$; below this curve $D>1$ and 


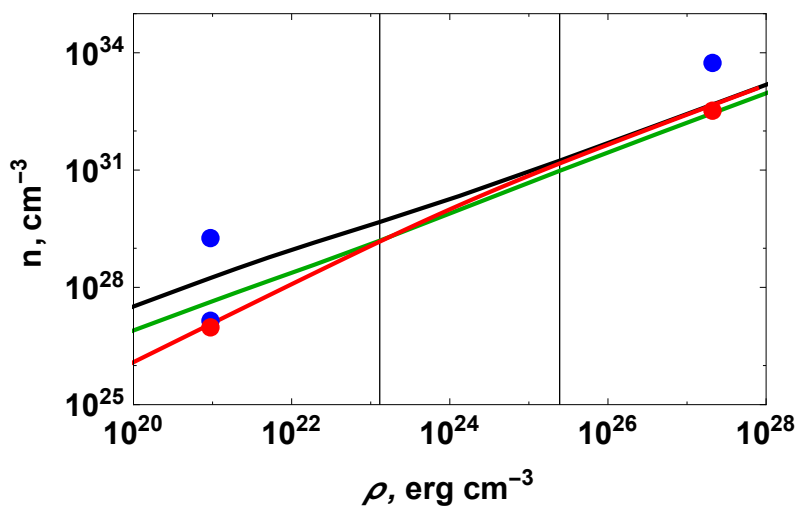

FIG. 1: Number density - energy density diagram of a photonelectron-positron plasma. Green curve corresponds to thermal equilibrium state. Black curve shows the transition from nondegenerate $D>1$ to degenerate $D<1$ plasma, where $D$ is defined by Eq. (1). Red curve corresponds to fully degenerate pair state defined by Eq. (2). Vertical line on the left corresponds to the transition from nonrelativistic to relativistic pair plasma $\left(\theta_{f i n}=0.3\right)$. Vertical line on the right corresponds to relativistic pair plasma with $\theta_{f i n}=1$. (Initial conditions for calculations are denoted by dots for pairs (red) and photons (blue).

plasma is nondegenerate, while above this curve $D<1$ and plasma is degenerate. Green curve corresponds to thermal equilibrium state. Red curve shows the maximum number density for electron-positron pairs (fully degenerate state). Note that thermal equilibrium state is very near to the border $D=1$.

Regarding fermions only (electrons and positrons without photons) it is important to note that both fully degenerate and thermal states have comparable number density of particles for ultrarelativistic average energy per particle (see Fig. 2, bottom), specifically number density for fully degenerate state is

$$
n_{c r}=\frac{8 \pi}{3 h^{3} c^{3}} \varepsilon_{F}^{3}
$$

and number density in ultrarelativistic limit for thermal state is

$$
n_{t h}=\frac{12 \pi \zeta(3)}{h^{3} c^{3}}(k T)^{3},
$$

where $\varepsilon_{F}$ is the Fermi energy, which plays a role of an upper particle energy boundary.

Occupation number of pairs $\langle n\rangle=h^{3} f / g$, where $f$ is distribution function, $g$ is the number of helicity states is shown in Fig. 2 (top) for selected temperatures, along with the corresponding spectral energy density $d \rho / d \epsilon$, see Fig. 2 (bottom). It is clear that for $\varepsilon \ll k T$ in thermal state $\langle n\rangle \simeq 1 / 2$.

To compare $n_{c r}$ with $n_{t h}$ we solve equation $\rho_{c r}=\rho_{t h}$ for $\varepsilon_{F}$, so we obtain $n_{c r}$ as a function of total energy density $\rho$ (or final equilibrium temperature $\theta$ ). On Fig. 3 we show the ratio $n_{c r} / n_{t h}$ as computed from the integral over the distribution function (red) and as computed

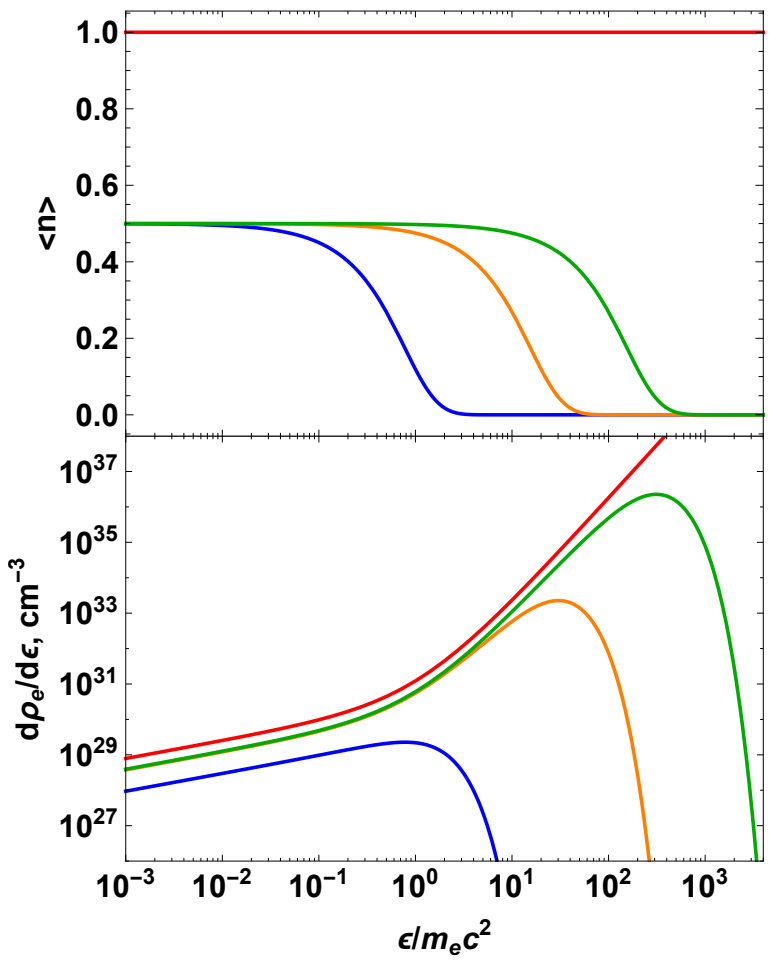

FIG. 2: Thermal average occupation numbers (top) and thermal spectral energy density (bottom) of pairs for selected temperatures: $\theta=0.5$ (blue), $\theta=10$ (orange), $\theta=100$ (green). The limiting spectral density for pairs according to Pauli principle is shown in red.

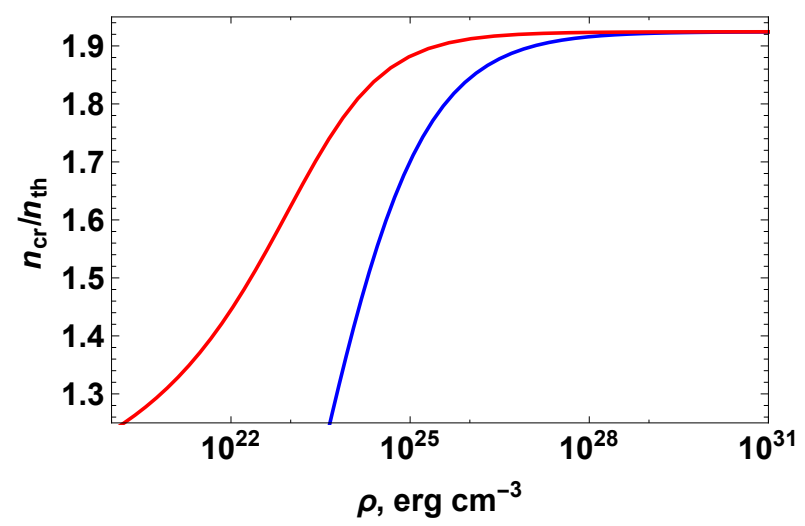

FIG. 3: The ratio $n_{c r} / n_{t h}$ computed numerically (red) and analytically in ultrarelativistic approximation (blue).

from ultrarelativistic expressions, eq. (3) and (2) (blue).

The relation $n_{c r} / n_{t h}$ is limited with high energy asymptotic $\frac{2^{1 / 4} 7^{3 / 4} \pi^{3}}{9 \times 15^{3 / 4} \zeta(3)} \simeq 1.9$. The last result shows that photonless plasma state cannot have $D \ll 1$. In other words, highly degenerate plasma should contain large number of photons in addition to pairs. 


\section{RELATIVISTIC BOLTZMANN EQUATIONS}

In order to study the influence of quantum degeneracy on thermalization process of electron-positron-photon plasma one should solve relativistic Boltzmann equations with Pauli blocking and Bose enhancement factors in collision integrals (Uehling-Uhlenbeck equations). In this work we focus on highly degenerate pair state and the effect of the Pauli blocking on plasma thermalization. Time evolution of one-particle distribution functions of electrons $e^{-}$, positrons $e^{+}$and photons $\gamma$ are found by numerical integration of relativistic Boltzmann equations [29] including quantum corrections

$$
\frac{1}{c} \frac{\partial f_{i}}{\partial t}=\sum_{q}\left(\eta_{i}^{q}-\chi_{i}^{q} f_{i}\right)
$$

where $f_{i}(\epsilon, t)$ are their distribution functions, index $i$ denotes the sort of particles, $\epsilon$ is their energy, $\eta_{i}^{q}$ and $\chi_{i}^{q}$ are the emission and the absorption coefficients of a particle of type " $i$ " via the physical process labelled by $q, c$ is the speed of light. The emission and absorption coefficients for the particle $I$ in a binary process $I+I I \leftrightarrows I I I+I V$ have the following form:

$$
\begin{aligned}
\eta_{I}^{2 p}= & \int d^{3} p_{2} d^{3} p_{3} d^{3} p_{4} W_{(3,4 \mid 1,2)} f_{I I I} f_{I V} \\
\times & \left(1+\xi f_{I}\right)\left(1+\xi f_{I I}\right), \\
\chi_{I}^{2 p} f_{I} & =\int d^{3} p_{2} d^{3} p_{3} d^{3} p_{4} W_{(1,2 \mid 3,4)} f_{I} f_{I I} \\
& \times\left(1+\xi f_{I I I}\right)\left(1+\xi f_{I V}\right),
\end{aligned}
$$

where transition rates are $W_{(3,4 \mid 1,2)} d^{3} p_{3} d^{3} p_{4}=$ $V d w_{(3,4 \mid 1,2)}$ and $W_{(1,2 \mid 3,4)} d^{3} p_{1} d^{3} p_{2}=V d w_{(1,2 \mid 3,4)}$, $V$ is normalization volume, $d w$ is differential reaction probability per unit time, $\xi=\psi h^{3} / 2$ and $\psi$ is $+1,-1,0$ for Bose-Einstein, Fermi-Dirac, Maxwell-Boltzmann statistic, respectively. In what follows we refer to these cases as quantum $(\psi= \pm 1)$ and classical $(\psi=0)$, respectively, $h$ is Planck's constant.

The emission and absorption coefficients for the particle $I$ in a triple process $I+I I \leftrightarrows I I I+I V+V$ have the following form:

$$
\begin{aligned}
\eta_{I}^{3 p}=\int d^{3} p_{2} d^{3} p_{3} d^{3} p_{4} d^{3} p_{5} W_{(3,4,5 \mid 1,2)} f_{I I I} f_{I V} f_{V} \\
\times\left(1+\xi f_{I}\right)\left(1+\xi f_{I I}\right), \\
\chi_{I}^{3 p} f_{I}=\int d^{3} p_{2} d^{3} p_{3} d^{3} p_{4} d^{3} p_{5} W_{(1,2 \mid 3,4,5)} \\
\quad \times f_{I} f_{I I}\left(1+\xi f_{I I I}\right)\left(1+\xi f_{I V}\right)\left(1+\xi f_{V}\right),
\end{aligned}
$$

where $W_{(3,4,5 \mid 1,2)} d^{3} p_{3} d^{3} p_{4} d^{3} p_{5}=V d w_{(3,4,5 \mid 1,2)}$ and $W_{(1,2 \mid 3,4,5)} d^{3} p_{1} d^{3} p_{2}=V^{2} d w_{(1,2 \mid 3,4,5)}$. The expression for

\begin{tabular}{|c|c|}
\hline Binary processes & Triple processes \\
\hline \hline Møller, Bhabha & Bremsstrahlung \\
$e^{ \pm} e^{ \pm \prime} \leftrightarrow e^{ \pm \prime \prime} e^{ \pm \prime \prime}$ & $e^{ \pm} e^{ \pm \prime} \leftrightarrow e^{ \pm \prime \prime} e^{ \pm \prime \prime \prime} \gamma$ \\
$e^{ \pm} e^{\mp} \leftrightarrow e^{ \pm \prime} e^{\mp^{\prime}}$ & $e^{ \pm} e^{\mp} \leftrightarrow e^{ \pm \prime} e^{\mp \prime} \gamma$ \\
\hline Single Compton & Double Compton \\
$e^{ \pm} \gamma \leftrightarrow e^{ \pm} \gamma^{\prime}$ & $e^{ \pm} \gamma \leftrightarrow e^{ \pm \prime} \gamma^{\prime} \gamma^{\prime \prime}$ \\
\hline Pair production & Radiative pair production, \\
and annihilation & triplet production \\
& and three photon annihilation \\
$\gamma \gamma^{\prime} \leftrightarrow e^{ \pm} e^{\mp}$ & $\gamma \gamma^{\prime} \leftrightarrow e^{ \pm} e^{\mp} \gamma^{\prime \prime}$ \\
& $e^{ \pm} \gamma \leftrightarrow e^{ \pm \prime} e^{\mp} e^{ \pm \prime \prime}$ \\
& $e^{ \pm} e^{\mp} \leftrightarrow \gamma \gamma^{\prime} \gamma^{\prime \prime}$ \\
\hline
\end{tabular}

TABLE I: Binary and triple QED processes in the pair plasma.

$d w$ is given in QED as:

$$
\begin{aligned}
d w & =c(2 \pi \hbar)^{4} \delta\left(\epsilon_{i n}-\epsilon_{f i n}\right) \delta\left(\mathbf{p}_{\text {in }}-\mathbf{p}_{\text {fin }}\right)\left|M_{f i}\right|^{2} V \\
& \times\left(\prod_{i n} \frac{\hbar c}{2 \epsilon_{i n} V}\right)\left(\prod_{f i n} \frac{d^{3} p_{f i n}}{(2 \pi \hbar)^{3}} \frac{\hbar c}{2 \epsilon_{f i n}}\right),
\end{aligned}
$$

where $\mathbf{p}_{\text {fin }}$ and $\epsilon_{\text {fin }}$ are respectively momenta and energies of outgoing particles, $\mathbf{p}_{i n}$ and $\epsilon_{i n}$ are momenta and energies of incoming particles, $M_{f i}$ is the corresponding matrix element, $\delta$-functions stand for energy-momentum conservation, $\hbar=h / 2 \pi$. Therefore, collision integrals, i.e. right-hand side of eqs. (4), are integrals over the phase space of interacting particles, which include the QED matrix elements, see e.g. [29, 31] for binary reactions and 32 for double Compton scattering, 33] for relativistic bremsstrahlung and 34] for substitution rules in computation of remaining matrix elements for triple reactions. We consider all binary and triple interactions between electrons, positrons and photons as listed in Tab. I]

Details of numerical integration scheme which solves the coupled system of integro-differential equations (4) on the grid in the phase space using a finite difference method is presented in 35 .

\section{THERMALIZATION OF SUPERDEGENERATE PLASMA}

Thermalization process with quantum degeneracy both in non-relativistic and relativistic cases was investigated in 36. There the relative role of binary and triple interactions has been studied. The only case with strongly degenerate conditions considered in that work was the case with the dominance of photons both in energy and in number. In this work we are interested in thermalization process of pair plasma with initially strongly degenerate distribution of electronpositron pairs. In addition, we require that initial conditions correspond to $D \ll 1$. Based on the results of the 


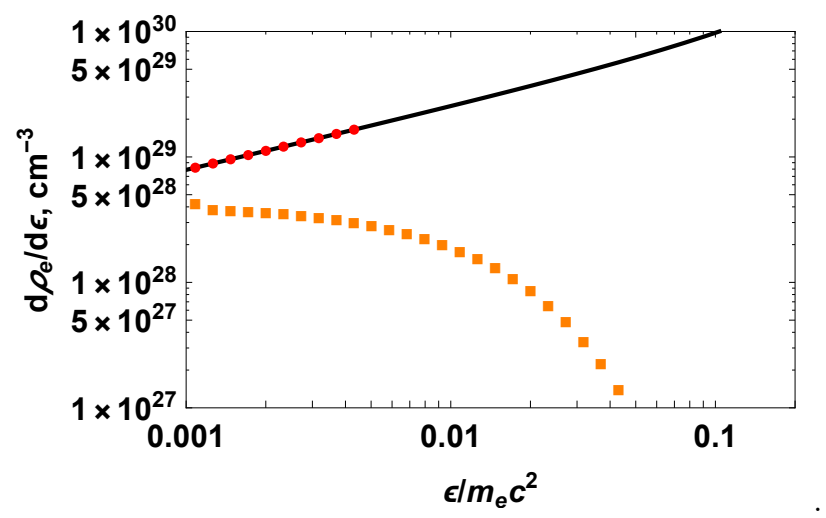

FIG. 4: Initial electron/positron spectral energy density: critical energy density with $\varepsilon_{F}=0.0043 m_{e} c^{2}$ (red), power law spectral energy density $d \rho / d \varepsilon=a\left(\varepsilon / \varepsilon_{0}\right)^{b}$, with $a=$ $2.778 \mathrm{~cm}^{-3}$ and $b=-81, \varepsilon_{0}=10^{-5.74}$ erg (orange). Horizontal axis shows kinetic energy without electron rest energy $m_{e} c^{2}$.

previous section, the contribution of pairs correspond to red dots in Fig. 1. It is clear that the density of photons in such a state should be larger than the density of pairs, and energy density of photons should be less than energy of pairs. The latter condition arises because the contribution of photons to the total energy density should be minimized, while their density should be maximized. In what follows we analyze specific initial conditions, represented in Fig. 1 by blue and red dots. We shall call this state a superdegenerate state.

With the goal to have $D \ll 1$ in the initial state we choose initial photon distribution as a delta function at the lowest energy state on our finite numerical grid. The distribution of pairs is chosen to be at equilibrium with zero temperature and non-zero chemical potential equal to the Fermi energy. We note that total critical pair number can be obtained with some nonequilibrium spectrum, which does not represent a degenerate state. Such cases are presented on figures below by solid and dashed curves.

Below we discuss four characteristic cases of initial conditions indicated by blue dots in the number density energy density diagram in Fig. 1] nonrelativistic and relativistic plasma with $D>1$ and $D<1$. It is appropriate to differentiate nonrelativistic and relativistic plasma by means of its average particle energy in thermal equilibrium: in nonrelativistic case this quantity is less than electron rest energy $\left(\rho_{t o t}^{t h}<m_{e} c^{2} n_{t o t}^{t h}\right)$, and in relativistic case otherwise.

First, we show the result of the simulation for nonrelativistic pair plasma with total energy density $\rho_{\text {tot }}=$ $9.4 \times 10^{20} \mathrm{erg} \mathrm{cm}^{-3}$ corresponding to a final equilibrium temperature $\theta_{f i n}=0.1$. Total initial particle number density is $n_{t o t}^{i n}=45 n_{\text {tot }}^{\text {fin }}$, where $n_{\text {tot }}^{\text {fin }}=4.2 \times 10^{27} \mathrm{~cm}^{-3}$ is the final total particle number density in equilibrium. Two different initial spectral distribution of pairs are con-

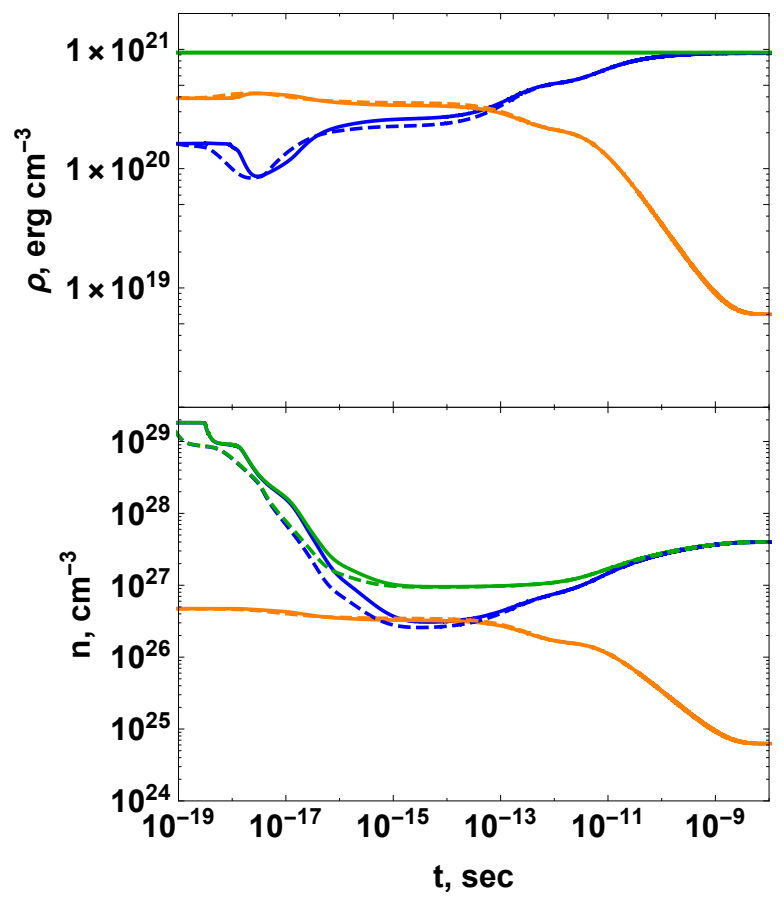

FIG. 5: Time evolution of energy density (top) and particle number density (bottom) for nonrelativistic pair plasma with degenerate initial pair state (solid) and nondegenerate initial pair state (dashed). Final equilibrium temperature is $\theta_{\text {fin }}=$ 0.1 .

sidered, see Fig. 44 a power law pair spectrum (orange), which is far from degenerate spectrum, or a fully degenerate state (red). The time evolution of basic thermodynamic quantities is shown on Fig. 5. Solid curves correspond to initial full degenerate pairs with zero temperature, while dashed curves correspond to non-equilibrium non-degenerate distribution of pairs. Total energy density does not change in time due to energy conservation. Total particle number density changes only due to imbalance in triple processes. The kinetic equilibrium is established at $t \simeq 2 \times 10^{-11} \mathrm{sec}$. The thermal equilibrium is reached with zero chemical potential and final temperature $\theta_{\text {fin }}=0.1$ at $t \simeq 10^{-8}$ sec. Spectral evolution of electrons is shown in Fig. 6. Note that pair annihilation is not subject to Pauli blocking so pair annihilation leads to disappearance of degeneracy. This result shows that thermalization process started from superdegenerate state is not influenced by quantum corrections and it is in a full agreement with the previous results obtained for the case of nondegenerate initial state [36. Thermalization dynamics in the case of non-equilibrium and non-degenerate distribution of pairs with the same particle energy density and particle number is presented by dashed curves in Fig. 5. Both solid and dashed curves show similar evolution.

Second, we turn to the case of electron-photon plasma. The positive charge, needed to compensate for electron 


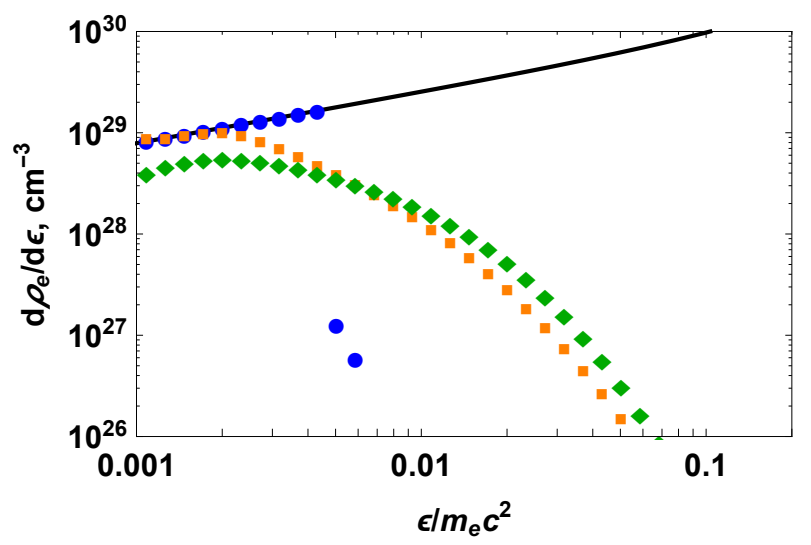

FIG. 6: Time evolution of electron/positron spectral energy density for nonrelativistic pair plasma with degenerate initial pair state for selected time moments: $t=1 \times 10^{-19}$ sec (blue), $t=8 \times 10^{-19} \mathrm{sec}$ (orange) and $t=9 \times 10^{-19} \mathrm{sec}$ (green). Final equilibrium temperature is $\theta_{f i n}=0.1$. Horizontal axis shows kinetic energy without electron rest energy $m_{e} c^{2}$.

charge, is assumed to be present, but its careers are not considered. The probability of creation of electronpositron pairs from photons at nonrelativistic temperature is exponentially suppressed. Then an initial fully degenerate electron state can be preserved for a time larger compared to the characteristic pair annihilation time. As a result, Pauli blocking multipliers might become important for thermalization process. In Fig. 7 we show the result of the simulation for the case of nonrelativistic photon-electron plasma with superdegenerate initial state (solid curves) and analogous simulation with nondegenerate initial electron state (dashed curves). Electron number is constant and photon number is changing due to imbalance in Double Compton scattering and Bremsstrahlung processes. There is a sharp difference between degenerate (solid curve) and non-degenerate (dashed curve) cases. For fully degenerate initial conditions Pauli blocking significantly reduces reaction rates (see Apendix). As a consequence kinetic evolution starts much later, only at $t \sim 10^{-15}$ sec with decrease of thermodynamic quantities. Then electron distribution quickly establishes the Fermi-Dirac form due to fast Coulomb scattering process. We note that photon state at that moment is not described yet by the Bose-Einstein distribution, because Compton scattering timescale is longer than Coulomb scattering one. The simulation shows that balance in Compton scattering process sets at the time moment $t \sim 6 \times 10^{-12} \mathrm{sec}$ and thermal equilibrium sets at $t \sim 10^{-11}$ sec. Thus, both binary and triple processes are unbalanced until final thermal equilibrium.

Third, we show the result of the simulation for relativistic pair plasma with total energy density $\rho_{\text {tot }}=$ $2.1 \times 10^{27} \mathrm{erg} \mathrm{cm}^{-3}$ corresponding to a final equilibrium temperature $\theta_{\text {fin }}=3$. Total initial particle number den-

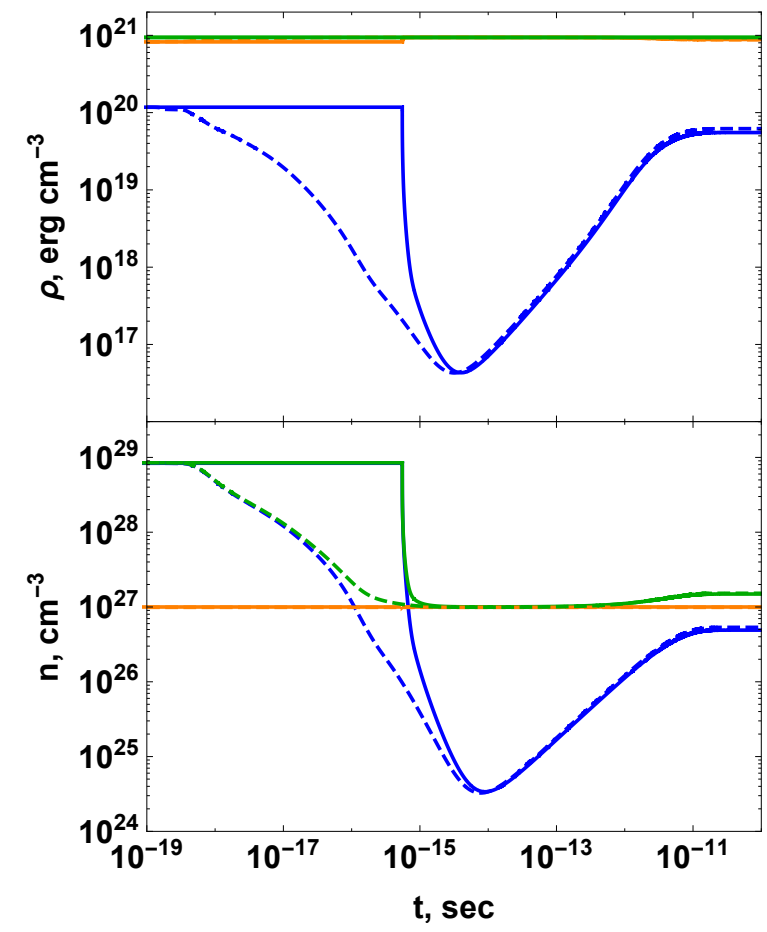

FIG. 7: Time evolution of energy density (top) and particle number density (bottom) for nonrelativistic photon-electron plasma with degenerate initial pair state (solid) and nondegenerate initial electron state (dashed). Final equilibrium temperature is $\theta_{\text {fin }}=0.05$.

sity is $n_{\text {tot }}^{i n}=20 n_{\text {tot }}^{f i n}$, where $n_{\text {tot }}^{f i n}=2.8 \times 10^{32} \mathrm{~cm}^{-3}$ is the final total particle number density in equilibrium. Time evolution of basic thermodynamic quantities is shown in Fig. 8. The kinetic equilibrium is absent in this case. The thermal equilibrium is reached at $t \sim 10^{-16}$ sec with zero chemical potential and final temperature $\theta_{f i n}=3$. As in the nonrelativistic case degenerate (solid curve) and non-degenerate (dashed curve) initial conditions lead to the similar thermalization process due to pair annihilation process. Thus, for the case of relativistic pair plasma thermalization process started from superdegenerate state is not influenced by Pauli blocking. Thermalization process goes in the same way as in the case of degenerate pairless initial state reported in [36]. Note that the faster onset of the evolution of the total particle number density of particles caused by the to Bose enhancement of two-photon annihilation rate in radiative pair production process, see Appendix, found previously is also valid for the case of superdegenerate initial conditions.

Finally, we discuss thermalization process with three different cases of relativistic photon-electron plasma. In contrast to nonrelativistic electron-photon plasma, relativistic plasma contains positrons. For a given total energy density the number of final positrons depends on the number of initial electrons. In equilibrium chem- 


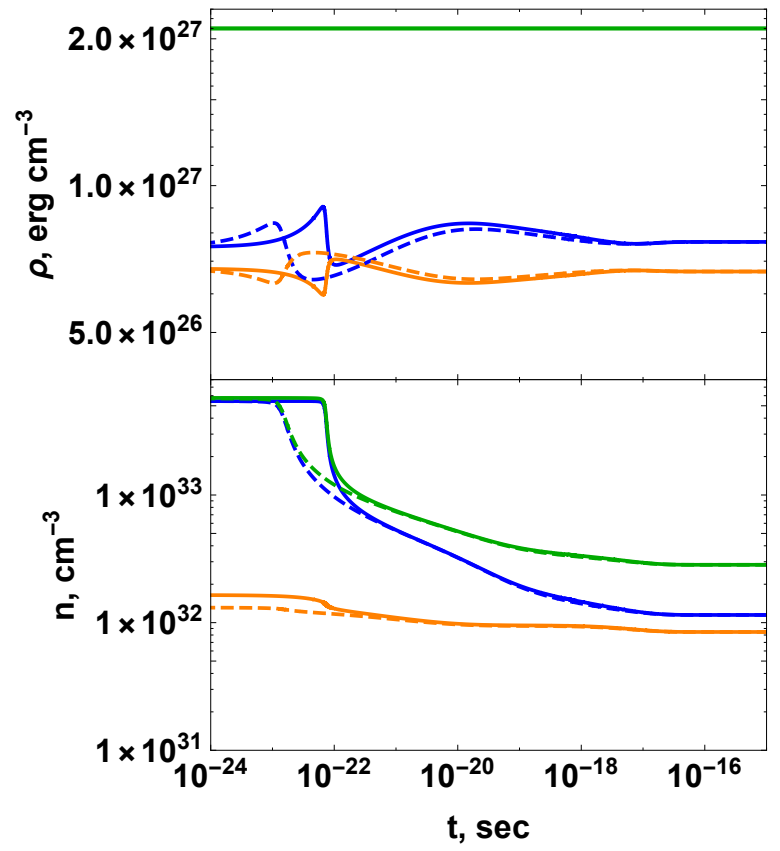

FIG. 8: Time evolution of energy density (top) and particle number density (bottom) for relativistic pair plasma with degenerate initial pair state (solid) and nondegenerate initial pair state (dashed). Final equilibrium temperature is $\theta_{\text {fin }}=3$.

ical potentials of electrons and positrons are equal on magnitude and their sum is zero $\left(\mu_{-}+\mu_{+}=0\right)$. If for the final state $\mu_{-}>1$ then final electron number is much greater than final positron number and plasma can be treated as electron-photon plasma. If for the final state $\mu_{-} \ll 1$ then final electron number is approximately equal to final positron number and plasma represents a transitional case between electron-photon plasma (when $\mu_{-}=-\mu_{+}>1$ ) and pair plasma (when $\left.\mu_{+}=\mu_{-}=0\right)$. First, the simulation for the relativistic transitional electron-photon plasma with fully degenerate initial electrons and $D \gg 1$ showed that thermalization for both degenerate and nondegenerate initial electrons proceeds in the similar way and Pauli blocking effects are negligible (see solid and dashed curves on Fig. 9p. The reason is that initial electron number is much less then final electron number $\left(n_{e}^{i n} \ll n_{e}^{f i n}\right)$ and the degree of electron degeneracy is not sufficient to block any reaction. This initial state is not a superdegenarate state because of violation of the condition $\rho_{e}^{i n}>\rho_{\gamma}^{i n}$. Second, for the initial state with a large number of degenerate electrons (when $n_{e}^{i n} \simeq n_{e}^{f i n}$ ) and with highly energetic photons (when $D \gtrsim 1$ ) the Pauli blocking effects are also negligible, because degeneracy of electrons vanishes due to Compton scattering (energetic photons scatter electrons to high energies). Third, we selected initial state containing a large number of degenerate electrons (when

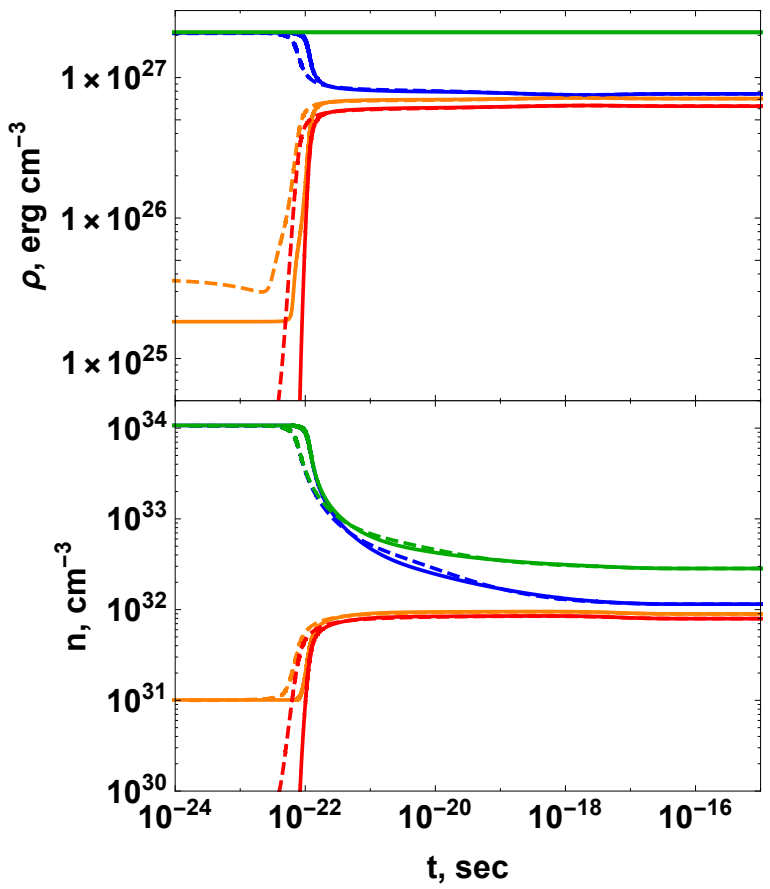

FIG. 9: Time evolution of energy density (top) and particle number density (bottom) for relativistic transitional electronphoton plasma with degenerate initial pair state (solid) and nondegenerate initial pair state (dashed). Final equilibrium temperature is $\theta_{\text {fin }}=3$.

$\left.n_{e}^{i n} \simeq n_{e}^{f i n}\right)$ and low energetic photons, that is the case $D \ll 1$ and present thermalization dynamics in Fig. 10 . As energy of initial photons is much less than $m c^{2}$, pair creation process is suppressed and initial degenerate electron state can be preserved until photons acquire energy more than $m c^{2}$. For the simulation the following initial conditions are chosen: total energy density is $\rho_{t o t}=$ $2.1 \times 10^{27} \mathrm{erg} \mathrm{cm}^{-3}$ corresponding to a final equilibrium temperature $\theta_{\text {fin }}=1.9$, total initial particle number density is $n_{\text {tot }}^{\text {in }}=40 n_{\text {tot }}^{\text {fin }}$, where $n_{\text {tot }}^{\text {fin }}=3.2 \times 10^{32} \mathrm{~cm}^{-3}$. One can see a sharp decrease of thermodynamic quantities at the time moment $t \sim 2 \times 10^{-19}$ sec, degenerate electron spectrum is preserved until the same time. The creation of positrons starts at the same moment. Thermalization process has an avalanche-like character. At the same time, simulation with nondegenerate initial electrons shows a smooth monotonic thermalization process (dashed curves in Fig. 10) which starts much earlier (solid curves on Fig. 10p.

\section{SUMMARY}

In this work the influence of Pauli blocking on thermalization process of relativistic plasma is studied for the first time using relativistic Uehling-Uhlenbeck equations taking into account all binary and triple QED pro- 


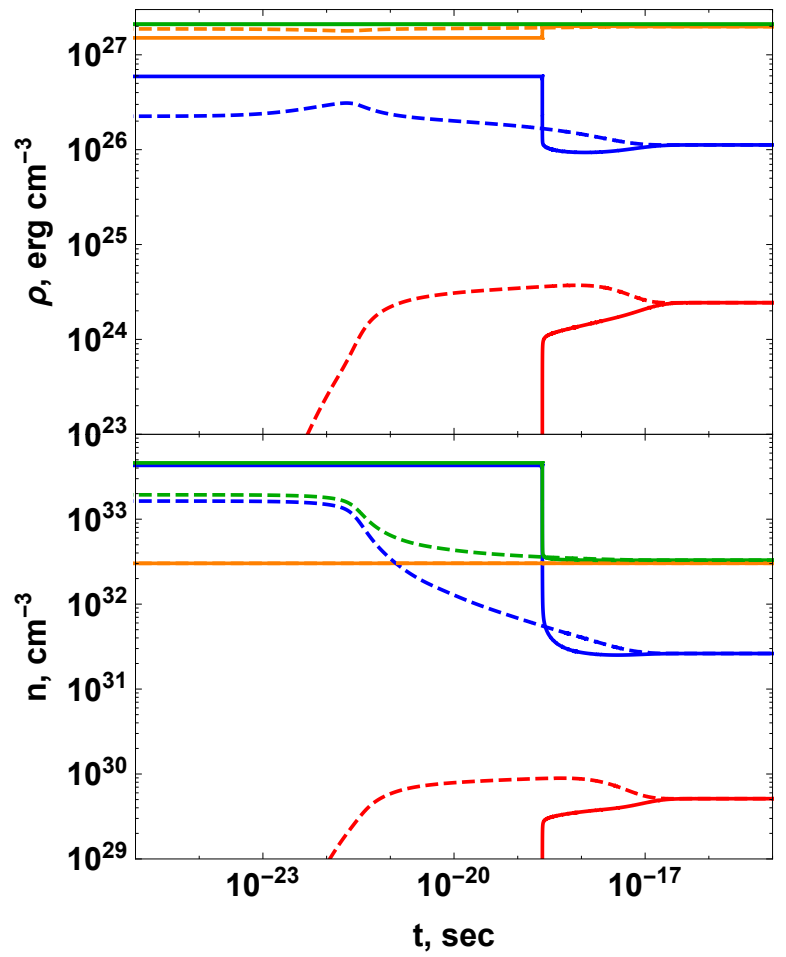

FIG. 10: Time evolution of energy density (top) and particle number density (bottom) for relativistic photon-electron plasma with degenerate initial pair state (solid) and nondegenerate initial electron state (dashed). Final equilibrium temperature is $\theta_{\text {fin }}=1.9$.

cesses. A wide range of initial conditions is analyzed, and several characteristic cases are presented. Both electronpositron-photon plasma, and photon-electron plasma are studied in relativistic and non relativistic domains.

We show that fully degenerate electron-positron state without photons corresponds to the degeneracy parameter $D \gtrsim 1$. Thus, in order study plasma evolution with $D \ll 1$ initial state should contain photons, in addition to degenerate electrons and positrons. The energy density and particle number density of plasma components in a such a superdegenerate state should satisfy the conditions: $\rho_{ \pm} \gg \rho_{\gamma}$ and $n_{ \pm} \ll n_{\gamma}$.
We found that thermalization process of superdegenerate pair plasma with total energy density ranging from $10^{20} \mathrm{erg} \mathrm{cm}^{-3}$ to $10^{28} \mathrm{erg} \mathrm{cm}^{-3}$ is not influenced by Pauli blocking and relaxation proceeds similarly both for $D \ll 1$ and for $D \gtrsim 1$. Pair annihilation process, not affected by the Pauli blocking, plays a crucial role here. This process quickly destroys initial degeneracy of electrons and positrons diminishing the role of the Pauli blocking effect.

At the same time, the process of thermalization in electron-photon plasma is very different. In nonrelativistic superdegenarate electron-photon plasma thermalization is delayed due to Pauli blocking of interactions with electrons. Once reactions are activated they have an avalanche-like behaviour. This is because initial state is preserved until photons can scatter degenerate electrons above their Fermi energy, where they states are not degenerate any more. The characteristic timescale for the beginning of the avalanche can be estimated as inverse reaction rate of the Compton scattering and it equals to $\tau_{\text {aval }} \simeq 10^{-15}$ sec for the number density $n_{\text {tot }}=8.5 \times 10^{28} \mathrm{~cm}^{-3}$. This is an interesting effect and it could manifest in laboratory and in astrophysical environments.

Relativistic electron-photon plasma contains positrons and initial conditions can be separated in two different classes defined by the conditions $\mu_{e}^{f i n} \ll 1$ and $\mu_{e}^{\text {fin }}>1$. In the first case final positron number density approximately equals to final electron number density. At the same time the number of initial degenerate electrons is not sufficiently large to satisfy the condition $\rho_{e}>\rho_{\gamma}$, and thermalization process of this type of relativistic electronphoton plasma is not affected by the Pauli blocking. For the second type of relativistic electron-photon plasma the superdegenerate state can exist and thermalization process in that case shows avalanche behaviour. The characteristic timescale for the beginning of avalanche is $\tau_{\text {aval }} \simeq 10^{-19} \mathrm{sec}$.

\section{Acknowledgments}

This work is supported within the joint BRFFRICRANet-2018 funding programme.

[1] W. Ebeling, D. Blaschke, R. Redmer, H. Reinholz, and G. Rpke, Journal of Physics A: Mathematical and Theoretical 42, 214033 (2009).

[2] G. M. Bruun, Few-Body Systems 45, 227 (2009).

[3] E. Wille et al., Phys. Rev. Lett. 100, 053201 (2008).

[4] S. Ospelkaus et al., Science 327, 853 (2010).

[5] L. De Marco et al., Science 363, 853 (2019).

[6] W. Ebeling, V. E. Fortov, and V. Filinov, Strongly interacting plasmas Quantum Statistics of Dense Gases and Nonideal Plasmas, Springer, 2017.

[7] M. Schmidt, T. Janke, and R. Redmer, Contributions to Plasma Physics 29, 431.

[8] G. Röpke et al., Phys. Rev. E 99, 033201 (2019).

[9] Y.-Z. Xing, H. Zhang, X.-B. Liu, and Y.-M. Zheng, Nuclear Physics A 957, 135 (2017). 


\begin{tabular}{|c|c|c|c|}
\hline & $\left(\gamma \gamma^{\prime} \leftrightarrow e^{ \pm} e^{\mp} \gamma^{\prime \prime}\right)$ & $e^{ \pm} \gamma \leftrightarrow e^{ \pm \prime} e^{\mp} e^{ \pm \prime \prime}$ & $e^{ \pm} \gamma \leftrightarrow e^{ \pm \prime} \gamma^{\prime} \gamma^{\prime \prime}$ \\
\hline$\eta_{\gamma}$ & $\begin{array}{l}f_{\gamma} f_{\gamma}\left(1+f_{\gamma}\right)\left(1-f_{+}\right)\left(1-f_{-}\right) \\
f_{\gamma} f_{+} f_{-}\left(1+f_{\gamma}\right)\left(1+f_{\gamma}\right)\end{array}$ & $\begin{array}{l}f_{ \pm} f_{+} f_{-}\left(1+f_{\gamma}\right)\left(1-f_{ \pm}\right) \\
f_{ \pm} f_{\gamma} f_{\gamma}\left(1-f_{ \pm}\right)\left(1+f_{\gamma}\right)\end{array}$ & $f_{\gamma} f_{ \pm}\left(1-f_{ \pm}\right)\left(1+f_{\gamma}\right)\left(1+f_{\gamma}\right)$ \\
\hline$\chi_{\gamma}$ & $\begin{array}{l}f_{\gamma} f_{+} f_{-}\left(1+f_{\gamma}\right)\left(1+f_{\gamma}\right) \\
f_{\gamma} f_{\gamma}\left(1+f_{\gamma}\right)\left(1-f_{+}\right)\left(1-f_{-}\right)\end{array}$ & $f_{\gamma} f_{ \pm}\left(1-f_{ \pm}\right)\left(1-f_{+}\right)\left(1-f_{-}\right)$ & $\begin{array}{l}f_{ \pm} f_{\gamma} f_{\gamma}\left(1-f_{ \pm}\right)\left(1+f_{\gamma}\right) \\
f_{\gamma} f_{ \pm}\left(1-f_{ \pm}\right)\left(1+f_{\gamma}\right)\left(1+f_{\gamma}\right)\end{array}$ \\
\hline$\eta_{ \pm}$ & $f_{\gamma} f_{\gamma}\left(1+f_{\gamma}\right)\left(1-f_{+}\right)\left(1-f_{-}\right)$ & $\begin{array}{l}f_{\gamma} f_{ \pm}\left(1-f_{ \pm}\right)\left(1-f_{+}\right)\left(1-f_{-}\right) \\
f_{ \pm} f_{+} f_{-}\left(1+f_{\gamma}\right)\left(1-f_{ \pm}\right)\end{array}$ & $\begin{array}{l}f_{\gamma} f_{ \pm}\left(1-f_{ \pm}\right)\left(1+f_{\gamma}\right)\left(1+f_{\gamma}\right) \\
f_{ \pm} f_{\gamma} f_{\gamma}\left(1-f_{ \pm}\right)\left(1+f_{\gamma}\right) \\
\end{array}$ \\
\hline$\chi_{ \pm}$ & $f_{\gamma} f_{+} f_{-}\left(1+f_{\gamma}\right)\left(1-f_{\gamma}\right)$ & $\begin{array}{l}f_{ \pm} f_{+} f_{-}\left(1+f_{\gamma}\right)\left(1-f_{ \pm}\right) \\
f_{\gamma} f_{ \pm}\left(1-f_{ \pm}\right)\left(1-f_{+}\right)\left(1-f_{-}\right)\end{array}$ & \begin{tabular}{|l|}
$f_{ \pm} f_{\gamma} f_{\gamma}\left(1-f_{ \pm}\right)\left(1+f_{\gamma}\right)$ \\
$f_{\gamma} f_{ \pm}\left(1-f_{ \pm}\right)\left(1+f_{\gamma}\right)\left(1+f_{\gamma}\right)$ \\
\end{tabular} \\
\hline & $e^{ \pm} e^{ \pm \prime} \leftrightarrow e^{ \pm \prime \prime} e^{ \pm \prime \prime \prime} \gamma$ & $e^{ \pm} e^{\mp} \leftrightarrow \gamma \gamma^{\prime} \gamma^{\prime \prime}$ & \\
\hline$\eta_{\gamma}$ & $f_{ \pm} f_{ \pm}\left(1-f_{ \pm}\right)\left(1-f_{ \pm}\right)\left(1+f_{\gamma}\right)$ & $f_{+} f_{-}\left(1+f_{\gamma}\right)\left(1+f_{\gamma}\right)\left(1+f_{\gamma}\right)$ & \\
\hline$\chi_{\gamma}$ & $f_{ \pm} f_{ \pm} f_{\gamma}\left(1-f_{ \pm}\right)\left(1-f_{ \pm}\right)$ & $f_{\gamma} f_{\gamma} f_{\gamma}\left(1-f_{+}\right)\left(1-f_{-}\right)$ & \\
\hline$\eta_{ \pm}$ & $f_{ \pm} f_{ \pm}\left(1-f_{ \pm}\right)\left(1-f_{ \pm}\right)\left(1+f_{\gamma}\right)$ & $\begin{array}{l}f_{\gamma} f_{ \pm}\left(1-f_{ \pm}\right)\left(1-f_{+}\right)\left(1-f_{-}\right) \\
f_{ \pm} f_{ \pm} f_{\gamma}\left(1-f_{ \pm}\right)\left(1-f_{ \pm}\right)\end{array}$ & \\
\hline$\chi_{ \pm}$ & $\begin{array}{l}f_{ \pm} f_{ \pm} f_{\gamma}\left(1-f_{ \pm}\right)\left(1-f_{ \pm}\right) \\
f_{ \pm} f_{ \pm}\left(1-f_{ \pm}\right)\left(1-f_{ \pm}\right)\left(1+f_{\gamma}\right)\end{array}$ & $f_{+} f_{-}\left(1+f_{\gamma}\right)\left(1+f_{\gamma}\right)\left(1+f_{\gamma}\right)$ & \\
\hline & $e^{ \pm} e^{\mp} \leftrightarrow \gamma \gamma^{\prime}$ & $e^{ \pm} \gamma \leftrightarrow e^{ \pm \prime} \gamma^{\prime}$ & $e^{ \pm} e^{ \pm} \leftrightarrow e^{ \pm \prime} e^{ \pm \prime}$ \\
\hline$\eta_{\gamma}$ & $f_{+} f_{-}\left(1+f_{\gamma}\right)\left(1+f_{\gamma}\right)$ & $f_{ \pm} f_{\gamma}\left(1-f_{ \pm}\right)\left(1+f_{\gamma}\right)$ & $f_{ \pm} f_{ \pm}\left(1-f_{ \pm}\right)\left(1-f_{ \pm}\right)$ \\
\hline$\chi_{\gamma}$ & $f_{\gamma} f_{\gamma}\left(1-f_{+}\right)\left(1-f_{-}\right)$ & $f_{ \pm} f_{\gamma}\left(1-f_{ \pm}\right)\left(1+f_{\gamma}\right)$ & $f_{ \pm} f_{ \pm}\left(1-f_{ \pm}\right)\left(1-f_{ \pm}\right)$ \\
\hline$\eta_{ \pm}$ & $f_{\gamma} f_{\gamma}\left(1-f_{+}\right)\left(1-f_{-}\right)$ & $f_{ \pm} f_{\gamma}\left(1-f_{ \pm}\right)\left(1+f_{\gamma}\right)$ & $f_{ \pm} f_{ \pm}\left(1-f_{ \pm}\right)\left(1-f_{ \pm}\right)$ \\
\hline$\chi_{ \pm}$ & $f_{+} f_{-}\left(1+f_{\gamma}\right)\left(1+f_{\gamma}\right)$ & $f_{ \pm} f_{\gamma}\left(1-f_{ \pm}\right)\left(1+f_{\gamma}\right)$ & $f_{ \pm} f_{ \pm}\left(1-f_{ \pm}\right)\left(1-f_{ \pm}\right)$ \\
\hline
\end{tabular}

TABLE II: Bose enhancement and Pauli blocking multipliers for collision integral of Uehling-Uhlenbeck equation for a given QED process. Here constant $h^{3} / 2$ is omitted.

[10] L. Ray, Phys. Rev. C 47, 2236 (1993).

[11] C. A. Bertulani and C. De Conti, Phys. Rev. C 81, 064603 (2010).

[12] A. M. Stefanini et al., Phys. Rev. C 100, 044619 (2019).

[13] B. DeMarco and D. S. Jin, Science 285, 1703 (1999).

[14] B. DeMarco, S. B. Papp, and D. S. Jin, Phys. Rev. Lett. 86, 5409 (2001).

[15] G. Ferrari, Phys. Rev. A 59, R4125 (1999).

[16] K. Aikawa et al., Phys. Rev. Lett. 112, 010404 (2014).

[17] A. Omran et al., Phys. Rev. Lett. 115, 263001 (2015).

[18] S. Chandrasekhar, The Astrophysical Journal 74, 81 (1931).

[19] S. Chandrasekhar, MNRAS 95, 207 (1935).

[20] N. K. Glendenning, Compact stars : nuclear physics, particle physics, and general relativity, Springer, 2000.

[21] R. Ruffini, G. V. Vereshchagin, and S.-S. Xue, Physics Reports 487, 1 (2010).

[22] C. S. Brady, C. P. Ridgers, T. D. Arber, and A. R. Bell, Physics of Plasmas 21, 033108 (2014).

[23] H. Chen et al., Physical review letters 114 (2015).

[24] A. Di Piazza, C. Müller, K. Z. Hatsagortsyan, and C. H. Keitel, Rev. Mod. Phys. 84, 1177 (2012).

[25] G. Sarri et al., Nat Commun 6, 6747 (2015).

[26] A. G. Aksenov, R. Ruffini, and G. V. Vereshchagin, Phys. Rev. Lett. 99, 125003 (2007).

[27] A. G. Aksenov, R. Ruffini, and G. V. Vereshchagin, Phys. Rev. D 79, 043008 (2009).

[28] A. G. Aksenov, R. Ruffini, and G. V. Vereshchagin, Phys. Rev. E81, 046401 (2010).

[29] G. V. Vereshchagin and A. G. Aksenov, Relativistic Kinetic Theory, Cambridge University Press, 2017.

[30] S. Groot, W. van Leeuwen, and C. van Weert, Relativistic Kinetic Theory: principles and applications, North-Holland Pub. Co., 1980.

[31] V. B. Berestetskii, E. M. Lifshitz, and V. B. Pitaevskii, Quantum Electrodynamics, Elsevier, 1982.

[32] F. Mandl and T. H. R. Skyrme, Proceedings of the Royal Society of London Series A 215, 497 (1952).

[33] E. Haug and W. Nakel, The Elementary Process of Bremsstrahlung, World Scientific Publishing Co, 2004.

[34] J. M. Jauch and F. Rohrlich, The Theory of Photons and Electrons, Springer-Verlag, 1976.

[35] M. A. Prakapenia, I. A. Siutsou, and G. V. Vereshchagin, Journal of Computational Physics 373,533 (2018).

[36] M. Prakapenia, I. Siutsou, and G. Vereshchagin, Physics Letters A 383, 306 (2019). 